\title{
EDITORIAL
}

\section{What Is the Role of Artificial Intelligence in Sports?}

\author{
Vasant Dhar \\ Editor-in-Chief
}

I attended an interesting set of talks at the most recent KDD conference in Halifax, Canada, on the use of data-driven analytics in sports. ${ }^{1,2}$ A couple of years ago, I supervised a student project with an NBA basketball team using box scores and other ancillary data for making decisions about how to match up against an opposing team, especially late in the season when game outcomes can be critical and coaches must conserve their resources. While play-by-play data existed, it seemed like a poor representation of the full richness associated with a possession, ignoring important movement details such as intent, opportunity, risk, execution, etc. I lamented that computers were unable to transform a raw video feed automatically into a structured database consisting of useful metrics such as "counter attacking speed" or "defensive fallback effectiveness," or "risk to reward ratio" associated with passes and ball possessions. That has changed, and the implications are serious.

With the rapid advances of artificial intelligence (AI) in perception, machines are able to watch and analyze games at a fine-grained level that is virtually impossible for humans to record and process. Machines can now track players and calculate useful metrics without the need for laborious human labeling. ${ }^{3}$ They can even estimate the effectiveness of every pass along multiple dimensions, such as the risk-to-potential reward ratio associated with a pass, the "pressure" being applied by the opposing team before the pass, and so on. The machine is also able to infer things, like the intent of each pass, automatically by analyzing the positions and velocities of all players on the field. It can also automatically label such passes as "completed," "intercepted," "scored," etc.

In short, we are on the verge of a great leap forward in automated feature engineering in sports, from where strategic and tactical insights using supervised machine learning methods are just a step away. By "strategic," I mean decisions on hiring and composition of teams that balance risk and reward-much like financial portfolios of assets selected to minimize their overall risk-to-return ratio. By "tactical," I mean actionable recommendations such as how to play against a specific adversary, possibly in real time.

While there is reason to believe that data and analytics could provide useful insights for coaches, players, and sports analysts, it has been a laborious exercise to code games and analyze them. Now that this genie is out of the bottle, what does it mean for the future of sports? In particular, what is the role of AI in sports? Physical enhancement using machines is legitimate in training but not in competition; should we expect the same rules to apply to cognitive enhancement in the future?

For coaches, big data-driven analytics has just upped the ante. Ignoring data is perilous. Imagine competing against an adversary whose computer has watched every game in the league and has, for example, discovered patterns of play that are unadvisable, risky, or effective under various playing conditions. Could coaches really ignore these new sources of insight, especially in a league where players' skills approach parity and coaching and strategy are the differentiators? Probably not. In other words, coaches and strategists are more likely to use "cognitive skill enhancement" tools in the future, driven by data.

For players this is great news, especially for improving their decision making skills. A major differentiator of players is their decision making ability under pressure. Some players may have a tendency to pass too early or hold on to the ball for too long, given their size and speed. Imagine a machine that conducts a post-mortem of every game and calculates playerand team-level decision making effectiveness based on its internal calculation of possible available options at any time. These calculations could be based, for example, on a ranking of what players could have been doing at the time versus what they did. Imagine 
a practice session where players' sensors not only record their movements as they do currently, but also use their field of vision and communicate possible passing options in real time! Just as it is common for players to use machines for physical enhancement during training, it will be hard for players to pass up on a personal cognitive skill enhancement assistant.

But what happens when physical robots have become as agile as humans? Advances in the analysis of human motion ${ }^{4}$ for creating more agile robots that can function in noisy domains ${ }^{5}$ and integrate real time feedback with a physics-based description of the world ${ }^{6}$ are still in their infancy. However, when we do have robust real-world physical robots, will their agility coupled with their informational advantage render them the real sports champions? One articulated goal by robotics researchers is that of an all-robot soccer team defeating the world champions by $2050 .^{7}$ When this happens, will humans compete mostly for fun, as in chess? Should such machines, and Elon Musk's envisioned hybrid cyberhumans of the future with "neural laces" and robotic implants then be disqualified from competition? Might such champion robots even be fun to watch?

\section{References}

1. Aoki RYS, Assuncao RM, Vaz de Melo POS. Luck is hard to beat: The difficulty of sports prediction. In: KDD-2017 Proceedings, August 2017.

2. Power P, Ruiz H, Wei X, Lucey P. Not all passes are created equal: Objectively measuring the risk and reward of passes in soccer from tracking data. In: KDD-2017 Proceedings, August 2017.

3. Le $H$, Carr P, Yue Y, Lucey P. 2017. Data-driven ghosting using deep imitation learning. In: MIT Sloan Sports Analytics Conference. Available online at www.sloansportsconference.com/wp-content/uploads/2017/ 02/1671-2.pdf

4. Plappert M, Mandery C, Asfour T. The KIT motion-language dataset. Big Data. 2016;4:236-252.

5. Mendoza JP, Simmons R, Veloso M. Detection of Subtle context-dependent model inaccuracies in high-dimensional robot domains. Big Data. 2016;4:269-285.

6. Ratliff N, Meier F, Kappler D, Schaal S.DOOMED: Direct Online Optimization of Modeling Errors in Dynamics. Big Data. 2016;4:253-268.

7. Palca J. Peter Stone can't get enough of robots playing soccer. 2014. Available online at www.npr.org/2014/01/15/262641016/peter-stonecant-get-enough-of-robots-playing-soccer

Cite this article as: Dhar V (2017) What is the role of artificial intelligence in sports? Big Data 5:3, 173-174, DOI: 10.1089/big.2017.29022.vdb. 